\title{
Investigation of Thermodynamic Properties of Zirconia Thin Films by Statistical Moment Method
}

\author{
Vu Van Hung1, Le Thi Thanh Huong'2, Dang Thanh Hai ${ }^{3}$ \\ ${ }^{1}$ University of Education, VNU Hanoi, Hanoi, Vietnam \\ ${ }^{2}$ Hai Phong University, Haiphong, Vietnam \\ ${ }^{3}$ Vietnam Education Publishing House, Hanoi, Vietnam \\ Email: bangvu57@yahoo.com
}

How to cite this paper: Van Hung, V. Huong, L.T.T. and Hai, D.T. (2018) Investigation of Thermodynamic Properties of Zirconia Thin Films by Statistical Moment Method. Materials Sciences and Applications, 9, 949-964.

https://doi.org/10.4236/msa.2018.912068

Received: October 23, 2018

Accepted: November 20, 2018

Published: November 23, 2018

Copyright $\odot 2018$ by authors and Scientific Research Publishing Inc. This work is licensed under the Creative Commons Attribution International License (CC BY 4.0).

http://creativecommons.org/licenses/by/4.0/

\begin{abstract}
The moment method in statistical (SMM) dynamics is used to study the thermodynamic quantities of $\mathrm{ZrO}_{2}$ thin films taking into account the anharmonicity effects of the lattice vibrations. The average lattice constant, thermal expansion coefficient and specific heats at the constant volume of $\mathrm{ZrO}_{2}$ thin films are calculated as a function of temperature, pressure and thickness of thin film. SMM calculations are performed using the Buckingham potential for the $\mathrm{ZrO}_{2}$ thin films. In the present study, the influence of temperature, pressure and the size on the thermodynamic quantities of $\mathrm{ZrO}_{2}$ thin film have been studied using three different interatomic potentials. We discuss temperature and thickness dependences of some thermodynamic quantities of $\mathrm{ZrO}_{2}$ thin films and we compare our calculated results with those of the experimental results.
\end{abstract}

\section{Keywords}

Thin Film, Zirconia, Lattice Constant, Thermal Expansion Coefficient, Specific Heats at the Constant Volume, Statistical Moment Method

\section{Introduction}

Oxide thin films are used for multi-layer optical coatings, and multi-layer optical thin film devices. Among oxide materials, pure and doped $\mathrm{CeO}_{2}$ and $\mathrm{ZrO}_{2}$ (bulk and thin films) have attracted more attention because of its desirable properties, such as high stability against mechanical abrasion, chemical attack and high temperatures [1] [2]. These oxide thin films have been prepared by some con- 
ventional methods. Many physical deposition techniques, such as pulsed laser deposition (PLD), magnetron sputtering, or molecular beam epitaxy (MBE), have been used produce $\mathrm{CeO}_{2}$ thin films. For instance, Gerblinger et al. [3] have obtained $\mathrm{CeO}_{2}$ thin films on $\mathrm{Al}_{2} \mathrm{O}_{3}$ substrate by sputter process, or the deposition of cerium dioxide thin films on Ni textured substrates by metallorganic chemical vapor deposition (MOCVD) has been reported [4] [5] [6]. Chemical vapor deposition (CVD) is a well-known technique for preparing thin films. For example, yttria doped $\mathrm{CeO}_{2}$ (YDC) thin films have been grown on yttria doped zirconia (YSZ) single substrates by vacuum vapor deposition and slurry painting method [7]. Different several chemical methods have also been applied to produce $\mathrm{CeO}_{2}$ (or $\mathrm{ZrO}_{2}$ ) thin films, such as sol-gel, aerosol-assisted MOCVD [8], mist microwave-plasma chemical vapour deposition (MPCVD) [9], atomic layer deposition (ALD) [10] and spray pyrolysis [11]. Recently, the structural and optical properties of $\mathrm{ZrO}_{2}$ thin films in relation to thermal annealing times, and properties of surface ceria-zirconia solid solution films were investigated [12] [13]. $\mathrm{ZrO}_{2}$ thin films were grown by thermal oxidation of metallic zirconium films deposited by sputtering of zirconium target by DC magnetron sputtering technique [14].

Recently, extensive studies of elastic and thermodynamic properties of oxide materials appear because of their important applications in high-frequency resonators. These materials are systematically fabricated by film deposition techniques in devices and their elastic constants are definitely required. Knowledge of mechanical and thermodynamic properties of these oxide thin films is essential to design Micro-Electro-Mechanical Systems (MEMS) devices. It is known that the size effect of phonon frequency is attributed to phonon confinement, surface pressure, or interfacial vibration effects. Therefore, understanding the size effect of elasticity and thermodynamic properties and their theoretical mechanism is important.

Most previous theoretical studies were concerned with the material properties of $\mathrm{ZrO}_{2}, \mathrm{CeO}_{2}$ bulk and thin films at absolute zero temperature or low temperature while temperature and pressure dependences of thermodynamic quantities have not been studied in detail. Recently, temperature and pressure dependences of thermodynamic and elastic properties of bulk cerium dioxide have been studied using the analytic statistical moment method (SMM) [15] [16] [17]. The purpose of the present article is to investigate the temperature, pressure and thickness dependences of some thermodynamic properties of $\mathrm{ZrO}_{2}$ thin films using the SMM.

\section{Theoretical Approach}

Theoretical explanations for the size effect are made by introducing the surface energy contribution in the continuum mechanics or the computational simulations reflecting the surface stress, or the surface relaxation influence. In this present research, the influence of the size effect on thermodynamic properties of zirconia thin film is studied by introducing the surface energy contribution in 
the free energy of zirconium and oxygen atoms of surface layers.

Let us consider an oxide free standing thin film $\mathrm{RO}_{2}$ has $n$ atomic layers of $R$ atom $(\mathrm{R}=\mathrm{Zr})$ and $(n-1)$ atomic layer $O$ with the thickness $\mathrm{d}$ as shown in Figure 1. We assume that the thin film consists of two zirconium surface-layers, two oxygen next surface-layers, $(n-3)$ oxygen internal-layers and $(n-2)$ zirconium internal-layers. $\quad N_{R}^{\text {sur }}, N_{O}^{\text {sur }}, N_{R}^{\text {int }}, N_{O}^{\text {int }}$ are the number of $R$ or $O$ atoms on surface-layers, next-surface-layers and internal-layers of this thin film, respectively. The thin film $\mathrm{RO}_{2}$ has the cubic fluorite structure, then $N_{R}^{s u r}=N_{R}^{\text {int }}=N_{01}$, $N_{O}^{\text {sur }}=N_{O}^{\text {int }}=N_{02}$, and $N_{01} / N_{02}=1 / 2$. Then, the potential energy of the surface-layers with $N_{R}^{s u r}$ atoms $R$, and $N_{O}^{\text {sur }}$ atoms $O$ can be written as

$$
U^{\text {sur }}=U_{R}^{\text {sur }}+U_{O}^{\text {sur }}=\frac{N_{R}^{\text {sur }}}{2} \sum_{i} \varphi_{i 0}^{R-s u r}\left(\left|r_{i}+u_{i}\right|\right)+\frac{N_{O}^{\text {sur }}}{2} \sum_{i} \varphi_{i 0}^{O-s u r}\left(\left|r_{i}+u_{i}\right|\right),
$$

here, $r_{i}$ is the equilibrium position of $i$-th atom, $u_{i}$ is its displacement of the $i$-th atom from the equilibrium position; $\varphi_{i 0}^{R-\text { sur }}, \varphi_{i 0}^{O-\text { sur }}$ are the effective interatomic potential between 0 -th $R$ atom and $i$-th atom, the 0 -th $O$ atom and $i$-th atom, respectively.

First, we expand the potential energy of the system in terms of the atomic (ionic) displacements $u_{i}$ of the atom $i$.

$$
\begin{aligned}
& U^{s u r}=U_{R}^{s u r}+U_{O}^{s u r}=\frac{N_{R}^{s u r}}{2} \sum_{i}\left\{\varphi_{i 0}^{R-s u r}\left(\left|r_{i}\right|\right)+\frac{1}{2} \sum_{\alpha, \beta}\left(\frac{\partial^{2} \varphi_{i 0}^{R-s u r}}{\partial u_{i \alpha} \partial u_{i \beta}}\right)_{e q} u_{i \alpha} u_{i \beta}\right. \\
& \left.+\frac{1}{6} \sum_{\alpha, \beta, \gamma}\left(\frac{\partial^{3} \varphi_{i 0}^{R-s u r}}{\partial u_{i \alpha} \partial u_{i \beta} \partial u_{i \gamma}}\right)_{e q} u_{i \alpha} u_{i \beta} u_{i \gamma}+\frac{1}{24} \sum_{\alpha, \beta, \gamma, \eta}\left(\frac{\partial^{4} \varphi_{i 0}^{R-s u r}}{\partial u_{i \alpha} \partial u_{i \beta} \partial u_{i \gamma} \partial u_{i \eta}}\right)_{e q} u_{i \alpha} u_{i \beta} u_{i \gamma} u_{i \eta}+\cdots\right\} \\
& +\frac{N_{O}^{s u r}}{2} \sum_{i}\left\{\varphi_{i 0}^{O-s u r}\left(\left|r_{i}\right|\right)+\frac{1}{2} \sum_{\alpha, \beta}\left(\frac{\partial^{2} \varphi_{i 0}^{O-s u r}}{\partial u_{i \alpha} \partial u_{i \beta}}\right)_{e q} u_{i \alpha} u_{i \beta}+\frac{1}{6} \sum_{\alpha, \beta, \gamma}\left(\frac{\partial^{3} \varphi_{i 0}^{O-s u r}}{\partial u_{i \alpha} \partial u_{i \beta} \partial u_{i \gamma}}\right)_{e q} u_{i \alpha} u_{i \beta} u_{i \gamma}\right. \\
& \left.+\frac{1}{24} \sum_{\alpha, \beta, \gamma, \eta}\left(\frac{\partial^{4} \varphi_{i 0}^{O-s u r}}{\partial u_{i \alpha} \partial u_{i \beta} \partial u_{i \gamma} \partial u_{i \eta}}\right)_{e q} u_{i \alpha} u_{i \beta} u_{i \gamma} u_{i \eta}+\cdots\right\},
\end{aligned}
$$

where $u_{i \alpha}$ denotes $\alpha$-Cartesian component of the atomic displacement of $i$-th atom, and the subscript eq means the quantities calculated at the equilibrium state.

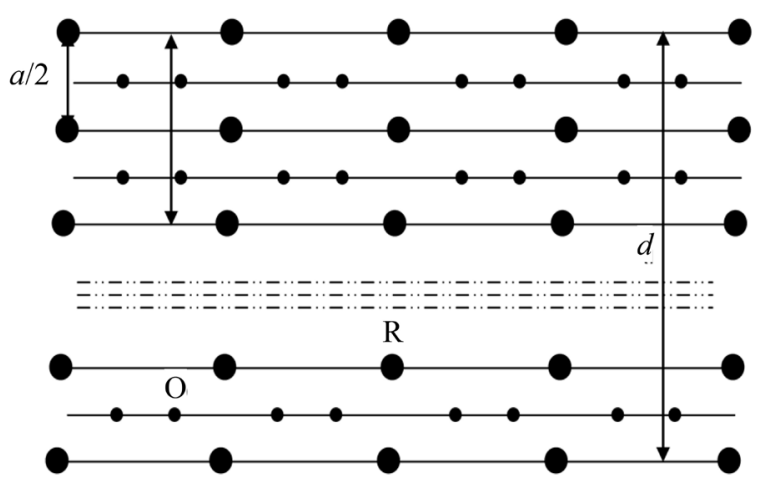

Figure 1. Free standing thin film $\mathrm{RO}_{2}$ with two $R$ surface-layers. 
Using Equation (2), the thermal average of the crystalline potential energy of the system is given in terms of the power moments $\left\langle u^{m}\right\rangle$ of the atomic displacements and the harmonic vibrational parameter $k$, and three anharmonic parameters $\beta, \gamma_{1}$ and $\gamma_{2}$ as

$$
\begin{aligned}
\left\langle U^{\text {sur }}\right\rangle= & U_{0}^{R-s u r}+U_{0}^{O-s u r}+3 N_{R}^{\text {sur }}\left[\frac{k_{R}^{\text {sur }}}{2}\left\langle\left(u_{R}^{\text {sur }}\right)^{2}\right\rangle+\gamma_{1}^{R-s u r}\left\langle\left(u_{R}^{\text {sur }}\right)^{4}\right\rangle\right. \\
& \left.+\gamma_{2}^{R-s u r}\left\langle\left(u_{R}^{\text {sur }}\right)^{2}\right\rangle^{2}\right]+3 N_{O}^{\text {sur }}\left[\frac{k_{O}^{\text {sur }}}{2}\left\langle\left(u_{O}^{\text {sur }}\right)^{2}\right\rangle+\beta_{O}^{\text {sur }}\left\langle u_{O}^{\text {sur }}\right\rangle\left\langle\left(u_{O}^{\text {sur }}\right)^{2}\right\rangle\right. \\
& \left.+\gamma_{1}^{O-s u r}\left\langle\left(u_{O}^{\text {sur }}\right)^{4}\right\rangle+\gamma_{2}^{O-\text { sur }}\left\langle\left(u_{O}^{\text {sur }}\right)^{2}\right\rangle^{2}\right]+\cdots,
\end{aligned}
$$

where

$$
\begin{gathered}
\gamma_{1}^{R-s u r}=\frac{1}{48} \sum_{i}\left(\frac{\partial^{4} \varphi_{i 0}^{R}}{\partial u_{i \alpha}^{4}}\right)_{e q}, \quad \gamma_{2}^{R-s u r}=\frac{6}{48} \sum_{i}\left(\frac{\partial^{4} \varphi_{i 0}^{R}}{\partial u_{i \alpha}^{2} \partial u_{i \beta}^{2}}\right)_{e q}, \\
\gamma_{1}^{O-s u r}=\frac{1}{48} \sum_{i}\left(\frac{\partial^{4} \varphi_{i 0}^{O}}{\partial u_{i \alpha}^{4}}\right)_{e q}, \quad \gamma_{2}^{O-s u r}=\frac{6}{48} \sum_{i}\left(\frac{\partial^{4} \varphi_{i 0}^{O}}{\partial u_{i \alpha}^{2} \partial u_{i \beta}^{2}}\right)_{e q}, \\
k_{R}^{s u r}=\frac{1}{2} \sum_{i}\left(\frac{\partial^{2} \varphi_{i 0}^{R}}{\partial u_{i \alpha}^{2}}\right)_{e q}, k_{O}^{s u r}=\frac{1}{2} \sum_{i}\left(\frac{\partial^{2} \varphi_{i 0}^{O}}{\partial u_{i \alpha}^{2}}\right)_{e q}, \\
\text { and } \beta_{O}^{s u r}=\frac{1}{2} \sum_{i}\left(\frac{\partial^{3} \varphi_{i 0}^{O}}{\partial u_{i \alpha} \partial u_{i \beta} \partial u_{i \gamma}}\right)_{e q},
\end{gathered}
$$

with $\alpha \neq \beta \neq \gamma=x, y$ or $z$. $U_{0}^{R-s u r}$ and $U_{0}^{O-s u r}$ represent the sum of effective pair interaction energies for $R$ and Oxygen atoms, respectively,

$$
\begin{aligned}
& U_{0}^{R-s u r}=\frac{N_{R}^{s u r}}{2} \sum_{i} \varphi_{i 0}^{R-s u r}\left(\left|r_{i}\right|\right), \\
& U_{0}^{O-\text { sur }}=\frac{N_{O}^{\text {sur }}}{2} \sum_{i} \varphi_{i 0}^{O-\text { sur }}\left(\left|r_{i}\right|\right),
\end{aligned}
$$

Let us consider a quantum system given by the following Hamiltonian:

$$
\hat{H}=\hat{H}_{0}-\sum_{i} \alpha_{i} \hat{V}_{L},
$$

where $\hat{H}_{0}$ denotes the lattice Hamiltonian in the harmonic approximation, and the second term $\sum_{i} \alpha_{i} \hat{V}_{L}$ is added due to the anharmonicity of thermal lattice vibrations, $\alpha_{i}$ denotes a parameter characterizing the anharmonicity of thermal lattice vibrations and $\hat{V}_{L}$ the related operator. The Helmholtz free energy of the system given by Hamiltonian (9) is formally written as [18] [19]

$$
\Psi=\Psi_{0}-\sum_{i} \int_{0}^{\alpha_{i}}\left\langle\hat{V}_{L}\right\rangle_{\alpha_{i}} \mathrm{~d} \alpha_{i},
$$

where $\left\langle\hat{V}_{L}\right\rangle_{\alpha_{i}}$ expresses the expectation value at the thermal equilibrium with the (anharmonic) Hamiltonian $\hat{H}$.

Using Equations (3), (9) and (10) permits us to calculate the Helmholtz free energy of $R$ atoms of the surface-layers as 


$$
\Psi_{R}^{s u r}=U_{0}^{R-s u r}+\Psi_{0}^{R-s u r}+\int_{0}^{\alpha_{i}}\left\langle V_{L}\right\rangle_{\alpha_{i}} \mathrm{~d} \alpha_{i}
$$

The Helmholtz free energy $\Psi_{R}^{\text {sur }}$ for $R$ atoms can be derived from the functional form of the potential energy of the above Equation (3) through the straightforward analytic integrations $I_{1}$ and $I_{2}$, with respect to the two anharmonicity "variables" $\gamma_{1}$ and $\gamma_{2}$. Firstly, for $R$ atoms $I_{1}$ and $I_{2}$ are written in an integral form as

$$
I_{1}=\int_{0}^{\gamma_{1}}\left\langle u^{4}\right\rangle \mathrm{d} \gamma_{1}, \quad I_{2}=\int_{0}^{\gamma_{2}}\left\langle u^{2}\right\rangle_{\gamma_{1}=0}^{2} \mathrm{~d} \gamma_{2}
$$

Then the free energy of the $N_{R}^{s u r}$ atoms $R$ of surface layers is given by

$$
\begin{aligned}
\Psi_{R}^{\text {sur }}= & U_{0}^{R-s u r}+\Psi_{0}^{R-s u r}+3 N_{R}^{\text {sur }} \int_{0}^{\gamma_{1}^{R-s u r}}\left\langle\left(u_{R}^{\text {sur }}\right)^{4}\right\rangle \mathrm{d} \gamma_{1}^{R-s u r} \\
& +3 N_{R}^{\text {sur }} \int_{0}^{\gamma_{2}^{R-s u r}}\left\langle\left(u_{R}^{\text {sur }}\right)^{2}\right\rangle_{\gamma_{1}^{R-s u r}=0}^{2} \mathrm{~d} \gamma_{2}^{R-s u r},
\end{aligned}
$$

where $\Psi_{0}^{R-s u r}$ denotes free energy in the harmonic approximation for the $N_{R}^{\text {sur }}$ atoms $R$ of surface layers which has the form as

$$
\Psi_{0}^{R-s u r}=3 N_{R}^{\text {sur }} \theta\left[x_{R}^{\text {sur }}+\ln \left(1-\mathrm{e}^{-2 x_{R}^{\text {sur }}}\right)\right] .
$$

Using the expression of the second and fourth moments [17] [20], we calculate the anharmonicity contribution to the free energy, then the free energy $\Psi_{0}^{R-s u r}$ of the surface layer atoms $R$ is given by

$$
\begin{aligned}
\Psi_{R}^{\text {sur }} \approx & \left\{U_{0}^{R-s u r}+\Psi_{0}^{R-s u r}\right\}+\frac{3 N_{R}^{\text {sur }} \theta^{2}}{\left(k_{R}^{\text {sur }}\right)^{2}}\left\{\gamma_{2}^{R-\text { sur }}\left(X_{R}^{\text {sur }}\right)^{2}-\frac{2 \gamma_{1}^{R-s u r}}{3}\left(1+\frac{X_{R}^{\text {sur }}}{2}\right)\right\} \\
& +\frac{6 N_{R}^{\text {sur }} \theta^{3}}{\left(k_{R}^{\text {sur }}\right)^{4}}\left\{\frac{4}{3}\left(\gamma_{2}^{R-\text { sur }}\right)^{2}\left(1+\frac{X_{R}^{\text {sur }}}{2}\right) X_{R}^{\text {sur }}\right. \\
& \left.-2\left(\left(\gamma_{1}^{R-\text { sur }}\right)^{2}+2 \gamma_{1}^{R-s u r} \gamma_{2}^{R-s u r}\right)\left(1+\frac{X_{R}^{\text {sur }}}{2}\right)\left(1+X_{R}^{\text {sur }}\right)\right\},
\end{aligned}
$$

where $x_{R}^{\text {sur }}=\frac{\hbar \omega_{R}^{\text {sur }}}{2 \theta}=\hbar \frac{\sqrt{k_{R}^{\text {sur }} / m}}{2 \theta}, X_{R}^{\text {sur }}=x_{R}^{\text {sur }} \operatorname{coth} x_{R}^{\text {sur }}$, and $m$ is the average atomic mass of the system, $m=C_{R} m_{R}+C_{O} m_{O}$.

Similar derivation can be also done for the free energy of $R$ and $O$ atoms of the surface-layers and internal-layers of thin film $\mathrm{RO}_{2}$. Free energies of these layer-types respectively are

$$
\begin{gathered}
\Psi_{O}^{\text {sur } \approx}=\left\{U_{0}^{O-\text { sur }}+\Psi_{0}^{O-\text { sur }}\right\}+\frac{3 N_{O}^{\text {sur }} \theta^{2}}{\left(k_{O}^{\text {sur }}\right)^{2}}\left\{\gamma_{2}^{O-\text { sur }}\left(X_{O}^{\text {sur }}\right)^{2}-\frac{2 \gamma_{1}^{O-\text { sur }}}{3}\left(1+\frac{X_{O}^{\text {sur }}}{2}\right)\right\} \\
+\frac{6 N_{O}^{\text {sur }} \theta^{3}}{\left(k_{O}^{\text {sur }}\right)^{4}}\left\{\frac{4}{3}\left(\gamma_{2}^{O-\text { sur }}\right)^{2}\left(1+\frac{X_{O}^{\text {sur }}}{2}\right) X_{O}^{\text {sur }}-2\left(\left(\gamma_{1}^{O-\text { sur }}\right)^{2}\right.\right.
\end{gathered}
$$




$$
\begin{aligned}
& \left.\left.+2 \gamma_{1}^{O-s u r} \cdot \gamma_{2}^{O-s u r}\right)\left(1+\frac{X_{O}^{\text {sur }}}{2}\right)\left(1+X_{O}^{\text {sur }}\right)\right\}+3 N_{O}^{\text {sur }} \theta\left[\frac{\left(\beta_{O}^{\text {sur }}\right)^{2}}{6 K_{\text {sur }}^{2}} \frac{k_{O}^{\text {sur }}}{\gamma_{0}^{O-s u r}}\right. \\
& \left.-\frac{\left(\beta_{O}^{\text {sur }}\right)^{2}}{6 K_{\text {sur }} \gamma_{0}^{O-\text { sur }}}\right]+3 N_{O}^{\text {sur }} \theta^{2}\left\{\frac{\beta_{O}^{\text {sur }}}{K_{\text {sur }}}\left[\frac{2 \gamma_{0}^{O-s u r}}{3 K_{\text {sur }}^{3}}\left(1+\frac{X_{O}^{\text {sur }}}{2}\right)^{1 / 2}\right]\right\} \\
& +3 N_{O}^{\text {sur }} \theta^{3}\left\{-\frac{\left(\beta_{O}^{\text {sur }}\right)^{2}}{9 K_{\text {sur }}^{3}}\left(1+\frac{X_{O}^{\text {sur }}}{2}\right)+\frac{\left(\beta_{O}^{\text {sur }}\right)^{2}}{9 K_{\text {sur }}^{4}}\left(1+X_{O}^{\text {sur }}\right)+\frac{\left(\beta_{O}^{\text {sur }}\right)^{2}}{6 K_{\text {sur }}^{2} k_{O}^{\text {sur }}}\left(X_{O}^{\text {sur }}-1\right)\right\} \text {, } \\
& \Psi_{R}^{i n t} \approx\left\{U_{0}^{R-i n t}+\Psi_{0}^{R-i n t}\right\}+\frac{3 N_{R}^{i n t} \theta^{2}}{\left(k_{R}^{i n t}\right)^{2}}\left\{\gamma_{2}^{R-i n t}\left(X_{R}^{i n t}\right)^{2}-\frac{2 \gamma_{1}^{R-i n t}}{3}\left(1+\frac{X_{R}^{i n t}}{2}\right)\right\} \\
& +\frac{6 N_{R}^{\text {int }} \theta^{3}}{\left(k_{R}^{i n t}\right)^{4}}\left\{\frac{4}{3}\left(\gamma_{2}^{R-i n t}\right)^{2}\left(1+\frac{X_{R}^{i n t}}{2}\right) X_{R}^{i n t}\right. \\
& \left.-2\left(\left(\gamma_{1}^{R-i n t}\right)^{2}+2 \gamma_{1}^{R-i n t} \cdot \gamma_{2}^{R-i n t}\right)\left(1+\frac{X_{R}^{i n t}}{2}\right)\left(1+X_{R}^{i n t}\right)\right\}, \\
& \Psi_{O}^{i n t} \approx\left\{U_{0}^{O-i n t}+\Psi_{0}^{O-i n t}\right\}+\frac{3 N_{O}^{i n t} \theta^{2}}{\left(k_{O}^{i n t}\right)^{2}}\left\{\gamma_{2}^{O-i n t}\left(X_{O}^{i n t}\right)^{2}-\frac{2 \gamma_{1}^{O-i n t}}{3}\left(1+\frac{X_{O}^{i n t}}{2}\right)\right\} \\
& +\frac{6 N_{O}^{i n t} \theta^{3}}{\left(k_{O}^{i n t}\right)^{4}}\left\{\frac{4}{3}\left(\gamma_{2}^{O-i n t}\right)^{2}\left(1+\frac{X_{O}^{i n t}}{2}\right) X_{O}^{i n t}-2\left(\left(\gamma_{1}^{O-i n t}\right)^{2}\right.\right. \\
& \left.\left.+2 \gamma_{1}^{O-i n t} \gamma_{2}^{O-i n t}\right)\left(1+\frac{X_{O}^{i n t}}{2}\right)\left(1+X_{O}^{i n t}\right)\right\}+3 N_{O}^{i n t} \theta\left[\frac{\left(\beta_{O}^{i n t}\right)^{2}}{6 K_{i n t}^{2}} \frac{k_{O}^{i n t}}{\gamma_{0}^{O-i n t}}\right. \\
& \left.-\frac{\left(\beta_{O}^{i n t}\right)^{2}}{6 K_{i n t} \gamma_{0}^{O-i n t}}\right]+3 N_{O}^{i n t} \theta^{2}\left\{\frac{\beta_{O}^{i n t}}{K_{\text {int }}}\left[\frac{2 \gamma_{0}^{O-i n t}}{3 K_{\text {int }}^{3}}\left(1+\frac{X_{O}^{\text {int }}}{2}\right)^{1 / 2}\right]\right\} \\
& +3 N_{O}^{i n t} \theta^{3}\left\{-\frac{\left(\beta_{O}^{i n t}\right)^{2}}{9 K_{i n t}^{3}}\left(1+\frac{X_{O}^{i n t}}{2}\right)+\frac{\left(\beta_{O}^{i n t}\right)^{2}}{9 K_{i n t}^{4}}\left(1+X_{O}^{i n t}\right)+\frac{\left(\beta_{O}^{i n t}\right)^{2}}{6 K_{i n t}^{2}} k_{O}^{i n t}\left(X_{O}^{i n t}-1\right)\right\},
\end{aligned}
$$

where

$$
\begin{gathered}
x_{O}^{\text {sur }}=\frac{\hbar \omega_{O}^{\text {sur }}}{2 \theta}=\hbar \frac{\sqrt{k_{O}^{\text {sur }} / m}}{2 \theta}, X_{O}^{\text {sur }}=x_{O}^{\text {sur }} \operatorname{coth} x_{O}^{\text {sur }}, \\
x_{R}^{\text {int }}=\frac{\hbar \omega_{R}^{\text {int }}}{2 \theta}=\hbar \frac{\sqrt{k_{R}^{\text {int }} / m}}{2 \theta}, X_{R}^{\text {int }}=x_{R}^{\text {int }} \operatorname{coth} x_{R}^{\text {int }}, \\
x_{O}^{\text {int }}=\frac{\hbar \omega_{O}^{\text {int }}}{2 \theta}=\hbar \frac{\sqrt{k_{O}^{\text {int }} / m}}{2 \theta}, X_{O}^{\text {int }}=x_{O}^{\text {int }} \operatorname{coth} x_{O}^{\text {int }},
\end{gathered}
$$

The number of atoms of internal-layers, and surface-layers of thin film $\mathrm{RO}_{2}$ are respectively determined as $N_{R}^{s u r}=N_{R}^{\text {int }}=N_{01}, \quad N_{O}^{s u r}=N_{O}^{\text {int }}=N_{02}$, and $N_{01} / N_{02}=1 / 2$. Free energy of thin film $R O_{2}$ and of one atom, respectively, are given by 


$$
\begin{aligned}
& \Psi=2 N_{01} \Psi_{R}^{s u r}+4 N_{01} \Psi_{O}^{s u r}+2(n-3) N_{01} \Psi_{O}^{\text {int }}+(n-2) N_{01} \Psi_{R}^{\text {int }}-T S_{c}, \\
& \frac{\Psi}{N}=\frac{2}{3 n-2} \Psi_{R}^{\text {sur }}+\frac{4}{3 n-2} \Psi_{O}^{\text {sur }}+\frac{2(n-3)}{3 n-2} \Psi_{O}^{\mathrm{int}}+\frac{n-2}{3 n-2} \Psi_{R}^{\mathrm{int}}-\frac{T S_{c}}{N},
\end{aligned}
$$

where $S_{c}$ is the configurational entropy of the system; $\Psi_{R}^{s u r}, \Psi_{O}^{s u r}, \Psi_{R}^{\text {int }}$ and $\Psi_{O}^{i n t}$ are correspondingly the free energy of one $R$ or $O$ atom at surface-layers and internal-layers.

Using $\bar{a}$ as the average nearest-neighbor distance (NND) and $b$ is the average thickness of two-layers and $\bar{a}_{h}$ is the average lattice constant. Then we have

$$
\bar{b}=\frac{2}{\sqrt{3}} \bar{a} ; \bar{a}_{h}=2 \bar{b}=\frac{4}{\sqrt{3}} \bar{a} .
$$

The average nearest-neighbor distance of thin film at a given temperature $T$ and pressure $P$ can be determined as

$$
\bar{a}(P, T)=\frac{2 a_{\text {sur }}(P, T)+(n-3) a_{\text {int }}(P, T)}{n-1} .
$$

The thickness $d$ of thin film can be given by

$$
\begin{gathered}
d=(n-1) \bar{b}=\frac{2(n-1)}{\sqrt{3}} \bar{a}, \\
\text { or } d=\frac{2}{\sqrt{3}}\left[2 a_{\text {sur }}(P, T)+(n-3) a_{\text {int }}(P, T)\right] .
\end{gathered}
$$

From Equations (21) and (23), we derived

$$
n=1+\frac{\sqrt{3}}{2 \bar{a}} d,
$$

and the average lattice constant of thin film $\mathrm{RO}_{2}$ is

$$
\bar{a}_{h}(P, T)=\frac{4}{\sqrt{3}} \cdot \frac{2 a_{\text {sur }}(P, T)+(n-3) a_{i n t}(P, T)}{n-1} .
$$

From Equation (26), one can find that the average lattice constant of the thin film depends on the thickness $\mathrm{d}$ of the thin film. When the number of crystalline layers $n$ is large enough, the thickness of the thin film reaches a certain limit, the average lattice constant approaches the value of lattice constant of the bulk material.

In above equation, $a_{\text {sur }}$ and $a_{\text {int }}$ are correspondingly the average NND between two intermediate atoms on the surface-layers, and internal-layers of thin film at a given temperature $T$ and pressure $P$. These quantities can be determined as

$$
\begin{gathered}
a_{\text {sur }}(P, T)=a_{\text {sur }}(P, 0)+C_{R} y_{0}^{R-s u r}(P, T)+C_{O} y_{0}^{O-\text { sur }}(P, T), \\
a_{\text {int }}(P, T)=a_{\text {int }}(P, 0)+C_{R} y_{0}^{R-\text { int }}(P, T)+C_{O} y_{0}^{O-\text { int }}(P, T),
\end{gathered}
$$

where $y_{0}^{R-s u r}(P, T), y_{0}^{O-s u r}(P, T), y_{0}^{R-i n t}(P, T), y_{0}^{O-i n t}(P, T)$ are the average displacements of $R$, and $O$ atoms from the equilibrium position in the surface-layers or internal-layers of thin film $\mathrm{RO}_{2}$ at given temperature $T$ and pressure $P$. The average displacements of $R$, and $O$ atoms $y_{0}^{R-s u r}(P, T), y_{0}^{O-s u r}(P, T), y_{0}^{R-i n t}(P, T), y_{0}^{O-i n t}(P, T)$ have the analytic forms as 
[19].

Substituting Equation (23) into Equation(20) we obtained the expression of the free energy per atom as follows

$$
\begin{aligned}
\frac{\Psi}{N}= & \frac{2}{1+\frac{3 \sqrt{3}}{2 \bar{a}} d} \Psi_{R}^{\text {sur }}+\frac{4}{1+\frac{3 \sqrt{3}}{2 \bar{a}} d} \Psi_{O}^{\text {sur }}+\frac{\frac{\sqrt{3}}{2 \bar{a}} d-1}{1+\frac{3 \sqrt{3}}{2 \bar{a}} d} \Psi_{R}^{\text {int }} \\
& +\frac{2\left(\frac{\sqrt{3}}{2 \bar{a}} d-2\right)}{1+\frac{3 \sqrt{3}}{2 \bar{a}} d} \Psi_{O}^{\text {int }}-\frac{T S_{c}}{N} .
\end{aligned}
$$

In the above Equations (15), (16), (17), and (18), the harmonic contributions to the Helmholtz free energies $\Psi_{0}^{R-s u r}, \Psi_{0}^{O-s u r}$ are derived using the "Einstein" approximation. The results of Equations (15)-(18) permits us to find the free energies $\Psi_{R}^{\text {sur }}, \Psi_{O}^{\text {sur }}$ at temperature $T$ under the condition that the parameters $k_{R, O}^{\text {sur }}, \gamma_{1}^{R, O-s u r}, \gamma_{2}^{R, O-s u r}, \gamma^{R, O-s u r}, \beta_{O}^{\text {sur }}$ and $K_{\text {sur }}$ at temperature $T_{0}$ (for example $T_{0}=0$ $K)$ are known. If the temperature $T_{0}$ is not far from $T$, then one can see that the vibration of a particle around a new equilibrium position (corresponding to $T_{0}$ ) is harmonic. Therefore, Equations (15) and (18) can be taken only to the second term, i.e.

$$
\begin{aligned}
& \Psi_{R}^{\text {sur }}=U_{0}^{R-s u r}+\Psi_{0}^{R-s u r}=3 N_{R}^{\text {sur }}\left\{\frac{1}{6} u_{0}^{R-s u r}+\theta\left[x_{R}^{\text {sur }}+\ln \left(1-\mathrm{e}^{-2 x_{R}^{\text {sur }}}\right)\right]\right\}, \\
& \Psi_{O}^{\text {sur }}=U_{0}^{O-\text { sur }}+\Psi_{0}^{O-\text { sur }}=3 N_{O}^{\text {sur }}\left\{\frac{1}{6} u_{0}^{O-\text { sur }}+\theta\left[x_{O}^{\text {sur }}+\ln \left(1-\mathrm{e}^{-2 x_{O}^{\text {sur }}}\right)\right]\right\},
\end{aligned}
$$

where

$$
\begin{aligned}
& u_{0}^{R-s u r}=\sum_{i} \varphi_{i 0}^{R-s u r}\left(\left|r_{i}\right|\right) \\
& u_{0}^{O-s u r}=\sum_{i} \varphi_{i 0}^{O-s u r}\left(\left|r_{i}\right|\right) .
\end{aligned}
$$

Since pressure $P$ is determined by

$$
P=-\left(\frac{\partial \Psi}{\partial V}\right)_{T}=-\frac{a}{3 V}\left(\frac{\partial \Psi}{\partial a}\right)_{T},
$$

from Equations (30), and (31), it is easy to take out an equation-of-states of a thin film system consists of one $R$ surface-layer, and one oxygen next surface-layer at zero temperature $T=0 \mathrm{~K}$ and pressure $P$

$$
P v=-a_{\text {sur }}\left\{C_{R}\left[\frac{1}{6} \frac{\partial u_{0}^{R-s u r}}{\partial a_{\text {sur }}}+\frac{\omega_{R}^{\text {sur }}(0)}{4 k_{R}^{\text {sur }}} \frac{\partial k_{R}^{\text {sur }}}{\partial a_{\text {sur }}}\right]+C_{O}\left[\frac{1}{6} \frac{\partial u_{0}^{O-\text { sur }}}{\partial a_{\text {sur }}}+\frac{\omega_{O}^{\text {sur }}(0)}{4 k_{O}^{\text {sur }}} \frac{\partial k_{O}^{\text {sur }}}{\partial a_{\text {sur }}}\right]\right\} \text {. }
$$

Similar derivation can be also done for the equation-of-states of internal-layers of thin film $R O_{2}$. The equation-of-states of these layer-types respectively are

$$
P v=-a_{i n t}\left\{C_{R}\left[\frac{1}{6} \frac{\partial u_{0}^{R-i n t}}{\partial a_{i n t}}+\frac{\omega_{R}^{i n t}(0)}{4 k_{R}^{i n t}} \frac{\partial k_{R}^{i n t}}{\partial a_{i n t}}\right]+C_{O}\left[\frac{1}{6} \frac{\partial u_{0}^{O-i n t}}{\partial a_{i n t}}+\frac{\omega_{O}^{i n t}(0)}{4 k_{O}^{i n t}} \frac{\partial k_{O}^{i n t}}{\partial a_{i n t}}\right]\right\} .
$$


In the above Equations (34) and (35), $\omega_{R}^{\text {sur }}(P, 0), \omega_{O}^{\text {sur }}(P, 0), \omega_{R}^{\text {int }}(P, 0)$ and $\omega_{O}^{\text {int }}(P, 0)$ are the vibration frequency of $R$ (or $\mathrm{O}$ ) atoms of surface-layers and internal-layers of thin film $R O_{2}$ at zero temperature $(T=0 \mathrm{~K})$ and pressure $P$.

With the aid of the free energy formula $\Psi=E-T S$, we can find the thermodynamic quantities of the system. Using Equation (29), it is easy to obtain the specific heats at constant volume $C_{V}$ of thin film $\mathrm{RO}_{2}$

$$
\begin{aligned}
C_{V}= & {\left[\frac{\partial E}{\partial T}\right]_{V}=-T \frac{\partial^{2} \Psi}{\partial T^{2}} } \\
= & \frac{2}{1+\frac{3 \sqrt{3}}{2 \bar{a}} d} C_{V}^{R-s u r}+\frac{4}{1+\frac{3 \sqrt{3}}{2 \bar{a}} d} C_{V}^{O-s u r} \\
& +\frac{\frac{\sqrt{3}}{2 \bar{a}} d-1}{1+\frac{3 \sqrt{3}}{2 \bar{a}} d} C_{V}^{R-i n t}+\frac{2\left(\frac{\sqrt{3}}{2 \bar{a}} d-2\right)}{1+\frac{3 \sqrt{3}}{2 \bar{a}} d} C_{V}^{O-i n t},
\end{aligned}
$$

where

$$
\begin{aligned}
& C_{V}^{R-s u r}=-T \frac{\partial^{2} \Psi_{R}^{\text {sur }}}{\partial T^{2}} ; C_{V}^{O-\text { sur }}=-T \frac{\partial^{2} \Psi_{O}^{\text {sur }}}{\partial T^{2}} ; \\
& C_{V}^{R-i n t}=-T \frac{\partial^{2} \Psi_{R}^{\text {int }}}{\partial T^{2}} ; C_{V}^{O-i n t}=-T \frac{\partial^{2} \Psi_{O}^{\text {int }}}{\partial T^{2}} .
\end{aligned}
$$

\section{Results and Discussion}

To calculate the thermodynamic quantities of $\mathrm{ZrO}_{2}$ thin film, we will use three different potentials, which include the electrostatic Coulomb interactions and two body terms to describe the short-range interactions. The two body terms arise from the electronic repulsion and attractive van der Waals forces, and they are described by a Buckingham potential form

$$
\varphi_{i j}=\frac{q_{i} q_{j}}{r}+A_{i j} \exp \left(-\frac{r}{B_{i j}}\right)-\frac{C_{i j}}{r^{6}},
$$

where $q_{i}$ and $q_{j}$ are the charges of the $i$-th and the $j$-th ions, $r$ is the distance between them and the parameters $A_{i j} B_{i j}$ and $C_{i j}$ are empirically determined by [21] [22] (listed in Table 1).

Firstly, we calculate the lattice parameters of $\mathrm{ZrO}_{2}$ thin films at zero temperature and $293 \mathrm{~K}$ (room temperature) using the three different potentials ( $P 1, P 2$ and L-C potentials). One can see in Figures 2-4 that the calculated lattice parameter increases with the increasing thickness. In the case of $\mathrm{ZrO}_{2}$ thin films with a thickness $d$ increases to about $100 \AA$ (or number of layers $n$ increases to 20), the lattice parameters of $\mathrm{ZrO}_{2}$ thin films are similar to those of the bulk $\mathrm{ZrO}_{2}$. The full-potential linearized augmented-plane-wave (FLAPW) $a b$ initio calculation of Jansen [23], based on the density functional theory in the local-density approximation (LDA), give $a_{0}=5.03 \AA$, while Hartre-Fock calculations (the CRYSTAL code) give $a_{0}=5.035 \AA$ (both at zero K). The density functional theory (DFT) 
within the plane-wave pseudopotential (PWP) [24] and RIP give $a_{0}=5.134 \AA$, and $a_{0}=5.162 \AA$. These results and the CRYSTAL calculation [25] are larger than the experimental values. Our SMM calculations give a lattice parameter $a_{0}=5.114 \AA$ (using potential $P 1$ ), $a_{0}=5.081 \AA$ (using $P 2$ ), $a_{0}=4.956 \AA$ (using L-C potential) at zero temperature and are in best agreement with the experimental values $a_{0}=$ $5.086 \AA$ [26] and FLAPW-DFT, LMTO and Hartree-Fock calculations.

The variation of the lattice parameter of $\mathrm{ZrO}_{2}$ thin films as a function of pressure in the present work by SMM calculations using potentials 1, 2 and L-C potential (as presented in Figure 5). One can see in Figures 2-6 that the lattice parameters calculated by using potentials 1 and 2 are very similar. The small difference between the two calculations simply comes from the difference in zirconium-oxygen interaction potentials, since the ionic Coulomb contribution and the oxygen-oxygen potential are the same for potentials 1 and 2 . The calculated lattice parameters of $\mathrm{ZrO}_{2}$ thin film by potentials 1 and 2 are almost identical, while the L-C potential gives somewhat smaller values, shifted upwards about $2 \%$ at wider temperature range of $300 \mathrm{~K}-2900 \mathrm{~K}$ and under pressure range of $0 \mathrm{GPa}$ - $50 \mathrm{GPa}$.

Temperature dependence of the lattice parameters of $\mathrm{ZrO}_{2}$ thin film with three potentials presented in Figure 6 and Figure 7. These Figures also shows the SMM calculations of lattice constants of $\mathrm{ZrO}_{2}$ thin film as an increasing function of temperature. Temperature dependence of the lattice parameters of $\mathrm{ZrO} 2$ thin film with different atomic layer numbers is similar for wider temperature range (see Figure 7). Our SMM theory predicts the lattice parameters of the cubic fluorite thin film $\mathrm{ZrO}_{2}$ to increase rapidly with temperature in agreement with those measured by experiments (for bulk $\mathrm{ZrO}_{2}$ ) [26] [27] [28].

In Figure 8, we compare the SMM results of thermal expansion coefficient of $\mathrm{ZrO}_{2}$ thin films with different atomic layer number $(n=5,10,15,20$ and 100) using potential $P 1$ at zero pressures with the experimental results (for bulk $\mathrm{ZrO}_{2}$ ) [29]. Temperature dependence of thermal expansion coefficient and specific heats at the constant volume of $\mathrm{ZrO}_{2}$ thin films with different atomic layer number are similar for wider temperature range of $300 \mathrm{~K}-2900 \mathrm{~K}$ (as shown in Figure 8 and Figure 10). One can see in Figures 8-10 that when the thickness $d$ of thin film increases to about $100 \AA$ (or number of layers $n$ increases to 20), thermal expansion coefficient and specific heats at the constant volume of $\mathrm{ZrO}_{2}$ thin films approach to the value of these thermodynamic quantities of the bulk material.

Table 1. The parameters of the Buckingham potential of $\mathrm{ZrO}_{2}$.

\begin{tabular}{ccccc}
\hline Interactions & $A(\mathrm{eV})$ & $B(\AA)$ & $C\left(\mathrm{eV} . \AA^{6}\right)$ & \\
\hline $\mathrm{O}^{2-}-\mathrm{O}^{2-}$ & 9547.96 & 0.2192 & 32.0 & Potential 1 \\
$\mathrm{Zr}^{4+}-\mathrm{O}^{2-}$ & 1502.11 & 0.3477 & 5.1 & \\
$\mathrm{O}^{2-}-\mathrm{O}^{2-}$ & 9547.96 & 0.224 & 32.0 & Potential 2 \\
$\mathrm{Zr}^{4+}-\mathrm{O}^{2-}$ & 1502.11 & 0.345 & 5.1 & \\
$\mathrm{O}^{2-}-\mathrm{O}^{2-}$ & 22.764 & 0.149 & 112.2 & L-C Potential \\
$\mathrm{Zr}^{4+}-\mathrm{O}^{2-}$ & 1453.8 & 0.35 & 0.0 & \\
\hline
\end{tabular}




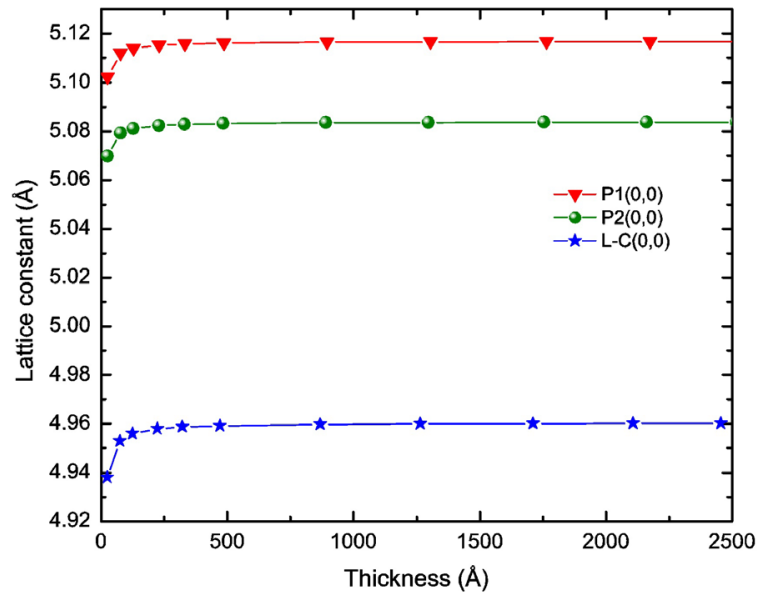

Figure 2. Thickness dependence of average lattice constant of thin film $\mathrm{ZrO}_{2}$ with Lewis-Catlow, $P 1$, and $P 2$ potentials at zero temperature and pressure $P=0 \mathrm{GPa}$.

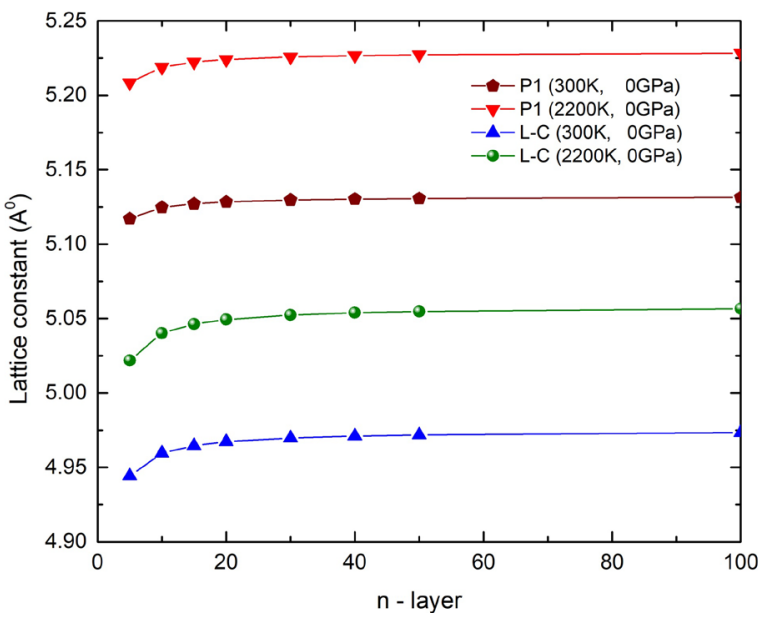

Figure 3. Atomic layers number dependence of average lattice constant of thin film $\mathrm{ZrO}_{2}$ with Lewis-Catlow, and $P 1$ potentials at zero pressure, and temperature $T=300 \mathrm{~K}$, and $2200 \mathrm{~K}$.

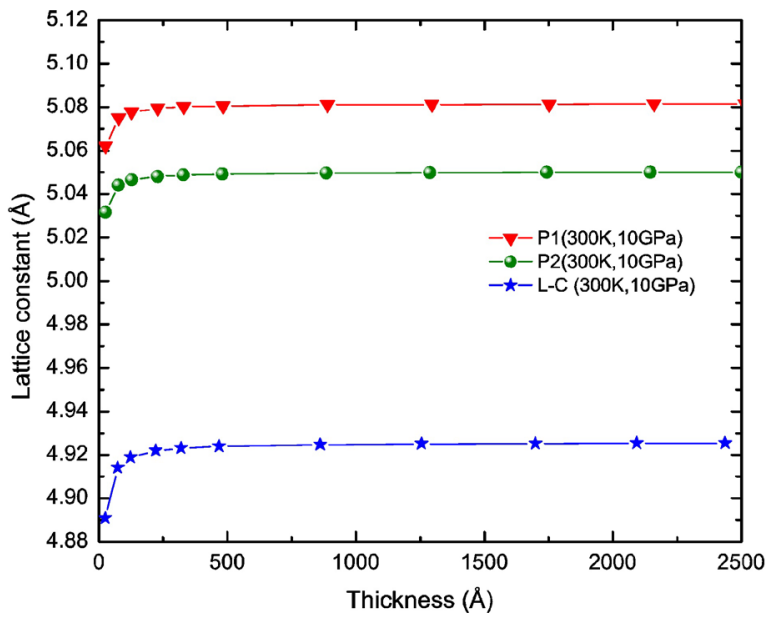

Figure 4. Thickness dependence of average lattice constant of thin film $\mathrm{ZrO}_{2}$ with Lewis-Catlow, $P 1$, and $P 2$ potentials at room temperature and pressure $P=10 \mathrm{GPa}$. 


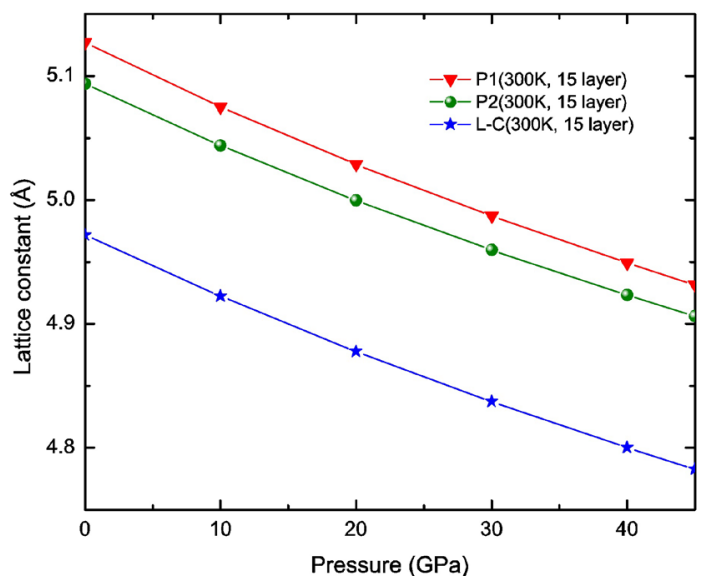

Figure 5. Pressure dependence of average lattice constant of $\mathrm{ZrO}_{2}$ thin film with atomic layer number $n=15$ at room temperature using Lewis-Catlow, $P 1$, an $P 2$ potentials.

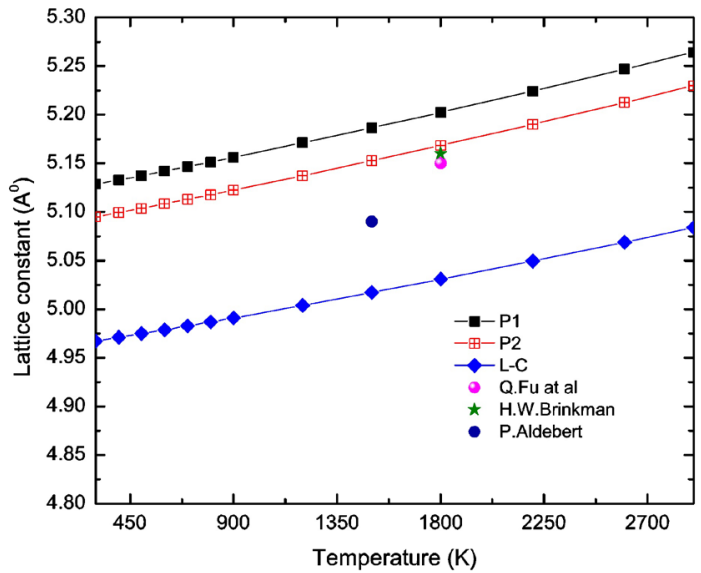

Figure 6. Temperature dependence of average lattice constant of $\mathrm{ZrO}_{2}$ thin film with atomic layer number $n=20$ at zero pressure $(P=0 \mathrm{GPa})$ using $P 1, P 2$ and Lewis-Catlow potentials. The red empty squares, black squares, and blue dimonds are the results from the SMM calculations, the royal and magenta circles and olive stars are the experimental results of bulk $\mathrm{ZrO}_{2}$ [26] [27] [28].

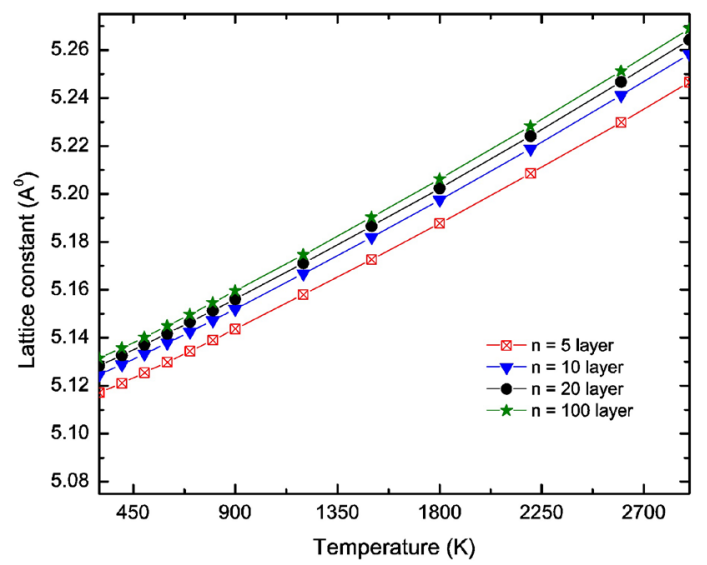

Figure 7. Temperature dependence of average lattice constant of $\mathrm{ZrO}_{2}$ thin film with different atomic layer numbers $(n=5,10,20$, and 100) at zero pressure $(P=0 \mathrm{GPa})$ using $P 1$ potential. 


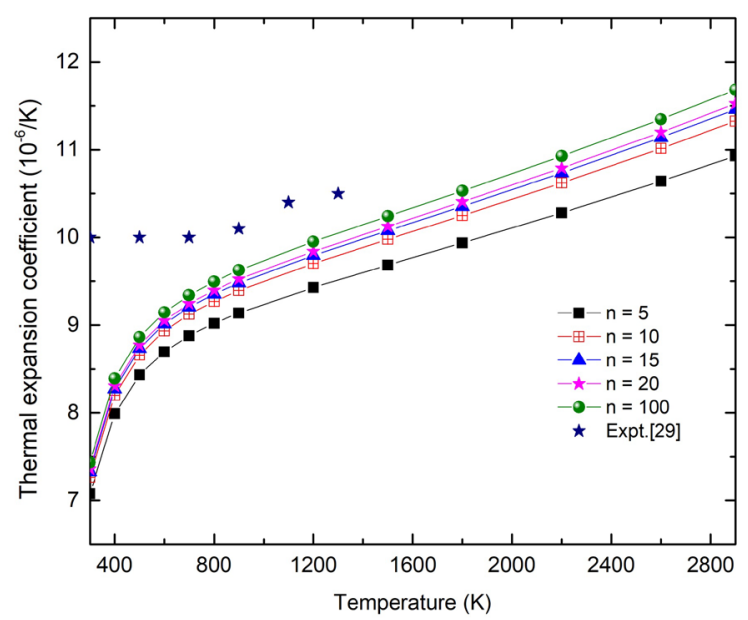

Figure 8. Temperature dependence of thermal expansion coefficient of $\mathrm{ZrO}_{2}$ thin films with different atomic layer numbers $(n=5,10,15,20$ and 100) at zero pressure using potential $P 1$. The navy stars are the experimental results of bulk $\mathrm{ZrO}_{2}$ [29].

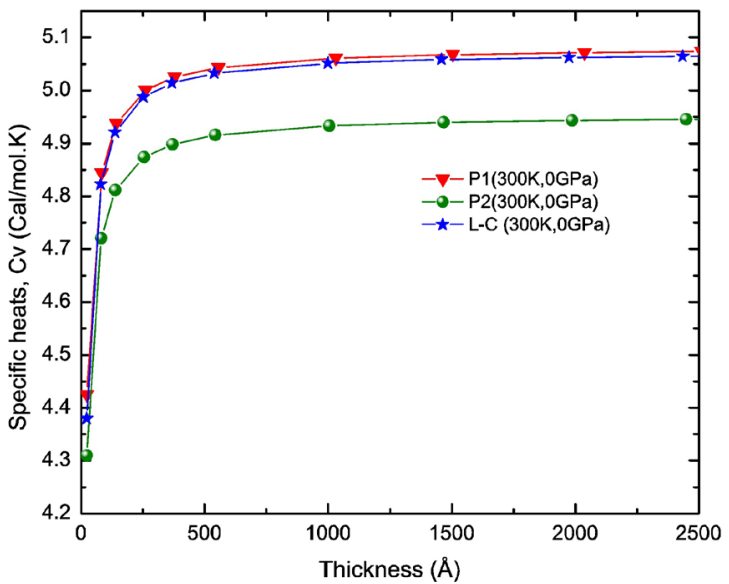

Figure 9. Thickness dependence of specific heats at the constant volume of $\mathrm{ZrO}_{2}$ thin film at room temperature and zero pressure using $P 1, P 2$ and Lewis-Catlow potentials.

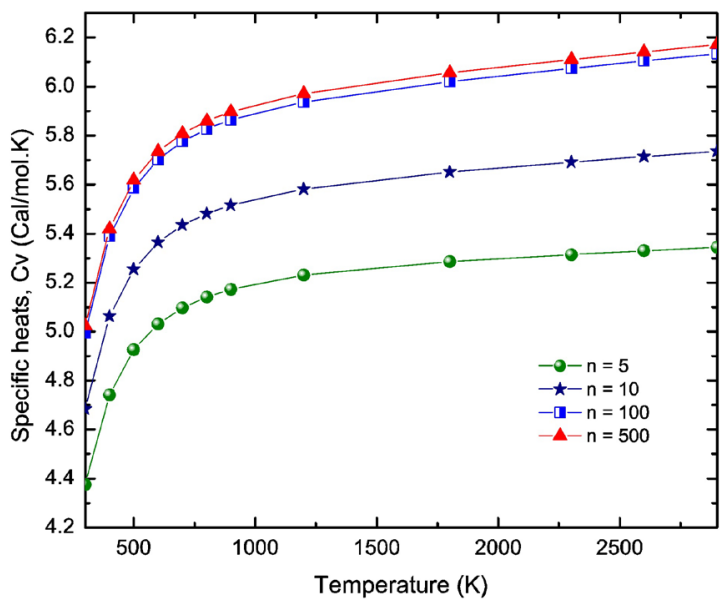

Figure 10. Temperature dependence of specific heats at the constant volume of $\mathrm{ZrO}_{2}$ thin films with different atomic layer numbers $(n=5,10,100$, and 500) at pressure $P=10 \mathrm{GPa}$ using potential $P$ l. 


\section{Conclusion}

In conclusion, it should be noted that the statistical moment method permits an investigation of the temperature, pressure and thickness dependences of $\mathrm{ZrO}_{2}$ thin films. The SMM calculations are performed by using Buckingham potential for $\mathrm{ZrO}_{2}$ thin films. Our development is establishing and solving equation of state to get the pressure dependence of the lattice constant, and then is the derivation of the analytical expressions of pressure dependence for some thermodynamic quantities of $\mathrm{ZrO}_{2}$ thin films as specific heats at the constant volume, as well as for the thermal expansion coefficient. The present formalism takes into account the higher-order anharmonic vibrational terms in the Helmholtz free energy of $\mathrm{ZrO}_{2}\left(\right.$ or $\left.\mathrm{CeO}_{2}\right)$ thin films and it enables us to derive the various thermodynamic quantities in closed analytic forms. Using the free energy formulas derived in the statistical moment method scheme, we have studied the temperature and thickness dependences of thermodynamic quantities of $\mathrm{ZrO}_{2}$ thin films. In general, we have found that the lattice constant, and thermal expansion coefficient of $\mathrm{ZrO}_{2}$ thin films are decreasing functions of the pressure, and increasing functions of the temperature and thickness. We have calculated thermodynamic quantities for $\mathrm{ZrO}_{2}$ thin films with different thickness using potentials 1 and 2 and Lewis-Catlow potential at various pressures, and these SMM calculated thermodynamic quantities of $\mathrm{ZrO}_{2}$ thin films with enough large layer number ( $n$ increases to 20) are in good agreement with the experiments of bulk $\mathrm{ZrO}_{2}$.

\section{Conflicts of Interest}

The authors declare no conflicts of interest regarding the publication of this paper.

\section{References}

[1] Farah, A.J., et al. (2009) Structural and Morphological Evolution of Cerium Oxide Thin Film on Silicon Prepared by Metal-Organic Decomposition Route. Journal of nuclear and Related Technologies, 6, 183-189.

[2] Rao K.N., Shivlingappa L. and Mohan S. (2003) Studies on Single Layer $\mathrm{CeO}_{2}$ and $\mathrm{SiO}_{2}$ Films Deposited by Rotating Crucible Electron Beam Evaporation. Materials Science and Engineering. B, 98, 38-44.

[3] Gerblinger, J., Lohwasser, W., Lampe, U. and Meixner, H. (1995) High Temperature Oxygen Sensor Based on Sputtered Cerium Oxide. Sensors and Actuators B: Chemical, 26, 93-96. https://doi.org/10.1016/0925-4005(94)01564-X

[4] Oh, S., Yoo, J., Lee, K., Kim, J.H. and Youm, D. (1998) Comparative Study on the Crack Formations in the CeO Buffer 2 Layers for YBCO Films on Textured Ni Tapes and Pt Tapes. Physica C: Superconductivity, 308, 91-98. https://doi.org/10.1016/S0921-4534(98)00412-2

[5] Kim, C.J., Kim, H.J., Sun, J.W., Ji, B.K., Kim, H.S., Joo, J., Jun, B.H., Jung, C.H., Park, S.D., Park, H.W. and Hong, G.W. (2003) Deposition of $\mathrm{CeO}_{2}$ and $\mathrm{NiO}$ Buffer Layers for YBCO Coated Conductors on Biaxially Textured Ni Substrates by a MOCVD Technique. Physica C: Superconductivity, 386, 327-332.

https://doi.org/10.1016/S0921-4534(02)02151-2 
[6] Graboy, I.E., Markov, N.V., Maleev, V.V., Kaul, A.R., Polyakov, S.N., Svetchnikov, L., Zandbergen, H.W. and Damhmen, K.H. (1997) An Improvement of Surface Smoothness and Lattice Match of $\mathrm{CeO}_{2}$ Buffer Layers on R-Sapphire Processed by MOCVD. Journal of Alloys and Compounds, 251, 318-321. https://doi.org/10.1016/S0925-8388(96)02700-4

[7] Horita, T., Sakai, N., Yokokawa, H., Dokiya, M., Kawada, T., Poulsen, F.W., et al. (1996) Electrochemical Reaction on Ceria Coated Zirconia in $\mathrm{H}_{2}-\mathrm{H}_{2} \mathrm{O}$ Atmosphere. Proceedings of the 17 th RISO International Symposium on Materials Science: High Temperature Electrochemistry: Ceramics and Metals, Roskilde, 2-6 September 1996, 281-286.

[8] Frohlich, K., Souc, J., Rosova, A., Machajdik, D., Graboy, I.E., Svetchnikov, V.L., Figueras, A. and Weiss, F. (1997) Superconducting Films Prepared by Aerosol Metal Organic Chemical Vapour Deposition on Substrate with Buffer Layer. Superconductor Science and Technology, 10, 657-662. https://doi.org/10.1088/0953-2048/10/9/005

[9] Takahashi, N., Koukitu, A. and Seki, H. (2001) Growth and Characterization of $\mathrm{YBa}_{2} \mathrm{Cu}_{3} \mathrm{O}_{x}$ and $\mathrm{NdBa}_{2} \mathrm{Cu}_{3} \mathrm{O}_{x}$ Superconducting Thin Films by Mist Microwave-Plasma Chemical Vapor Deposition Using a $\mathrm{CeO}_{2}$ Buffer Layer. Journal of Materials Science, 35, 1231-1238.

[10] Paivasaari, J., Putkonen, M. and Niinisto, L. (2002) Cerium Dioxide Buffer Layers at Low Temperature by Atomic Layer Deposition. Journal of Materials Chemistry, 12, 1828-1832. https://doi.org/10.1039/b108333c

[11] Elidrissi, B., Addou, M., Regragui, M., Monty, C., Bougrine, A. and Kachouane, A. (2000) Structural and Optical Properties of $\mathrm{CeO}_{2}$ Thin Films Prepared by Spray Pyrolysis. Thin Solid Films, 379, 23-27. https://doi.org/10.1016/S0040-6090(00)01404-8

[12] Onn, T.M., Mao, X., Lin, C., Wang, C. and Gorte, R.J. (2017) Investigation of the Thermodynamic Properties of Surface Ceria and Ceria-Zirconia Solid Solution Films Prepared by Atomic Layer Deposition on $\mathrm{Al}_{2} \mathrm{O}_{3}$. Inorganics, 5, 69-80. https://doi.org/10.3390/inorganics5040069

[13] Hojabri, A. (2016) Structural and Optical Characterization of $\mathrm{ZrO}_{2}$ Thin Films Grown on Silicon and Quartz Substrates. Journal of Theoretical and Applied Physics, 10, 219-224. https://doi.org/10.1007/s40094-016-0218-8

[14] Sunke, V. and Suda, U. (2018) Structural and Optical Properties of Thermally Oxidized Zirconium Dioxide Films. International Letters of Chemistry, Physics and Astronomy, 77, 15-25.

[15] Hung, V.V., Thanh, L.T.M. and Masuda-Jindo, K. (2010) Study of Thermodynamic Properties of Cerium Dioxide under High Pressures. Computational Materials Science, 49, 355-358. https://doi.org/10.1016/j.commatsci.2010.03.003

[16] Hung, V.V. and Thanh, L.T.M. (2011) Study of Elastic Properties of $\mathrm{CeO}_{2}$ by Statistical Moment Method. Physica B, 406, 4014-4018. https://doi.org/10.1016/j.physb.2011.07.012

[17] Hung, V.V., Lee, J. and Masuda-Jindo, K. (2006) Investigation of Thermodynamic Properties of Cerium Dioxide by Statistical Moment Method. Journal of Physics and Chemistry of Solids, 67, 682-689. https://doi.org/10.1016/j.jpcs.2005.09.100

[18] Hung, V.V., Hang, N.T. and Huong, L.T.T. (2012) Lattice Constant of Ceria Thin Film: Temperature Dependence. 57, 79.

[19] Hung, V.V., Thanh, L.T.M. and Hai, N.T. (2006) Investigation of Thermodynamic Quantities of the Cubic Zirconia by Statistical Moment Method. Advances in Natu- 
ral Sciences, 7, 21.

[20] Tang, N. and Hung, V.V. (1988) Investigation of the Thermodynamic Properties of Anharmonic Crystals by the Momentum Method. Physica Status Solidi (B), 149, 511-519. https://doi.org/10.1002/pssb.2221490212

[21] Houska, J. (2016) Force Field for Realistic Molecular Dynamics Simulations of $\mathrm{ZrO}_{2}$ Growth. Computational Materials Science, 111, 209. https://doi.org/10.1016/j.commatsci.2015.09.025

[22] Zacate, M.O., Minervini, L., Bradfield, D.J., Grimes, R.W. and Sickafus, K.E. (2000) Defect Cluster Formation in $\mathrm{M}_{2} \mathrm{O}_{3}$-Doped Cubic $\mathrm{ZrO}_{2}$. Solid State Ionics, 128, 245-254. https://doi.org/10.1016/S0167-2738(99)00348-3

[23] Jansen, H.I.F. (1991) Electronic Structure of Cubic and Tetragonal Zirconia. Physical Review B, 43, 7267-7278. https://doi.org/10.1103/PhysRevB.43.7267

[24] Walter, E.J., et al. (2001) First Principle Study of Carbon Monoxide Adsortion on Zirconia-Supported Copper. Surface Science, 495, 44-50. https://doi.org/10.1016/S0039-6028(01)01498-4

[25] Stefanovich, E.V., Shluger, A.L. and Catlow, C.R.A. (1994) Theoretical Study of the Stabilization of Cubic-Phase $\mathrm{ZrO}_{2}$ by Impurities. Physical Review B, 49, 11560-11571. https://doi.org/10.1103/PhysRevB.49.11560

[26] Aldebert, P. and Traverse, J.-P. (1985) Structure and Ionic Mobility of Zirconia at High Temperature. Journal of the American Ceramic Society, 68, 34-40. https://doi.org/10.1111/j.1151-2916.1985.tb15247.x

[27] Fu, Q., Saltsburg, H. and Flytzani-Stephanopoulos, M. (2003) Active Nonmetallic $\mathrm{Au}$ and Pt Species on Ceria-Based Water-Gas Shift Catalysts. Science, 301, 935-938. https://doi.org/10.1126/science.1085721

[28] Brinkman, H.W., Briels, W.J. and Verweij, H. (1995) Molecular Dynamics Simulation of Yttria-Stabilized Zirconia. Chemical Physics Letters, 247, 386-390. https://doi.org/10.1016/S0009-2614(95)01231-1

[29] Okur, A., Yigit, R., Celik, E. and Sayman, O. (2011) Thermal Stress Analysis in $\mathrm{ZrO}_{2}$ Insulation Coatings on Cr-Ni Substrates during Cooling Process. Mathematical and Computational Applications, 16, 598-604. https://doi.org/10.3390/mca16030598 